\section{Repercussões da amniorrexe prematura no pré-termo sobre a morbimortalidade neonatal}

\author{
Repercussions of premature rupture of fetal \\ membranes on neonatal morbidity and mortality
}

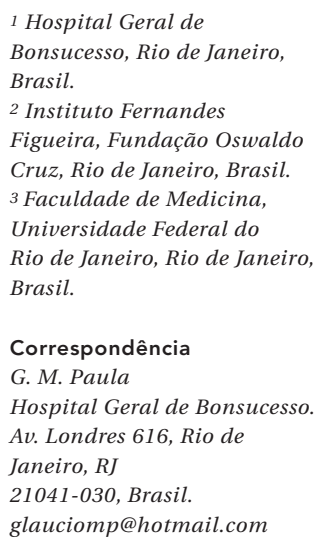

Correspondência G. M. Paula Hospital Geral de Bonsucesso. Av. Londres 616, Rio de Janeiro, $R J$

21041-030, Brasil.

glauciomp@hotmail.com

\begin{abstract}
The objective of this study was to analyze factors associated with death and survival with sequelae in neonates after premature rupture of fetal membranes (PROM). An observational historical cohort study analyzed charts of patients with PROM at $\leq 34$ weeks gestation. The variables were compared with neonatal death and survival with sequelae as the outcomes. In both groups, the data were submitted to bivariate analysis, and the variables showing significance were submitted to logistic regression. The final multivariate model for fetal death showed statistical significance for the following: chorioamnionitis; 5-minute Apgar score $\leq 5$; birth weight $\leq 1,000$; and cardiopulmonary resuscitation. Survival with sequela was associated with: cervical colonization; patent ductus arteriosus; 5-minute Apgar score $\leq$ 5; and birth weight $\leq 1,000 \mathrm{~g}$. Infections, very low birth weight, and peripartum asphyxia were the principal variables associated with the target outcomes among newborns from gestations involving PROM.
\end{abstract}

Premature Rupture Fetal Membranes; Neonatal Mortality (Public Health); Morbidity
Glaucio de Moraes Paula 1

Luiz Guilherme Pessoa da Silva 2,3

Maria Elizabeth Lopes Moreira 2

Olga Bonfim 2

\section{Introdução}

A ruptura prematura pré-termo das membranas amnióticas é uma complicação obstétrica observada em cerca de $3 \%$ das gestações e tem como principal repercussão o majorar das taxas de nascimentos prematuros, respondendo por até um terço destes 1 . O olhar atual sobre a amniorrexe remota ao termo e a entende como o produto final de uma complexa e multifatorial rede de processos patológicos que podem co-existir em uma mesma paciente.

A evidência da relação estreita entre processos infecciosos e/ou inflamatórios e a ocorrência de ruptura prematura pré-termo das membranas amnióticas parece bem fundamentada por diversos trabalhos, em que nem sempre é possível determinar se a ruptura das membranas ovulares levou ao processo infeccioso ou se foi este último o fator causal determinante da amniorrexe no pré-termo 2,3 .

Esse ambiente intra-uterino inóspito, com possível exposição fetal a processos inflamatórios e infecciosos, bem como ao nascimento prematuro, tornam a ruptura prematura pré-termo das membranas amnióticas uma importante causa de incremento na morbi-mortalidade neonatal e um grande desafio com o qual obstetras e neonatologistas se defrontam na atualidade 1 .

Os avanços tecnológicos e terapêuticos no campo neonatal nos trazem ainda uma nova questão a ser considerada que é a sobrevivência 
de recém-nascidos egressos de gestações que tiveram como intercorrência a ruptura prematura pré-termo das membranas amnióticas em idade gestacional muito prematura e com extremo baixo peso, agregando preocupações não somente concernentes à redução das taxas de mortalidade neonatal, mas também quanto à possibilidade de seqüelas com repercussões sobre a qualidade de vida e saúde destas crianças 2 .

Ao contrário das taxas de sobrevida que melhoraram muito nas últimas décadas, a incidência de morbidades nessa população específica de prematuros tem mostrado pouca alteração 4 . Os principais grupos de agravos associados à prematuridade e com factível possibilidade de comprometimento crônico compreendem: as lesões que incidem sobre o sistema nervoso central (principalmente a hemorragia intraventricular e leucomalacia periventricular), a displasia broncopulmonar e a retinopatia da prematuridade 5,6 .

Neste estudo procuramos com base em uma análise retrospectiva de gestantes que tiveram diagnóstico de ruptura das membranas amnióticas, avaliar a repercussão de fatores obstétricos e neonatais sobre a mortalidade neonatal e a ocorrência da seqüela entre os sobreviventes. Objetivamos trazer contribuição para o melhor conhecimento científico do tema em questão, colaborando assim para o aprofundamento das discussões sobre planejamento e administração de ações voltadas para a prevenção, abordagem terapêutica e reabilitação tanto no nível coletivo quanto individual.

\section{Metodologia}

Neste estudo transversal foram analisados casos de amniorrexe prematura em gestações pré-termo, considerando-se o recorte de 24 a 34 semanas de gestação no momento do parto, dados obtidos com base em prontuários médicos de pacientes internadas no Departamento de Obstetrícia e de Neonatologia do Instituto Fernandes Figueira (IFF), da Fundação Oswaldo Cruz, no período de 1988 a 2002.

Foram excluídos os casos de amniorrexe diagnosticados dentro do período aludido, mas que o parto tenha ocorrido em idade gestacional superior a 34 semanas exclusive, bem como os casos de gestações múltiplas e malformações congênitas.

O diagnóstico de amniorrexe foi pautado na visualização da saída de líquido amniótico pelo orifício cervical utilizando-se o exame especular, ou ainda pela confirmação por meio da realização de testes propedêuticos complementares, tais como: análise do $\mathrm{pH}$ vaginal com papel de nitrazina, cristalização com padrão arboriforme do líquido colhido no fundo de saco vaginal ou estudo citológico do referido líquido, a fim de pesquisar elementos celulares de origem fetal.

As variáveis estudadas foram divididas em clínico-obstétricas e neonatais. No primeiro grupo foram incluídas: a idade materna; a paridade; idade gestacional no momento do parto; infecção do trato urinário documentada pelo isolamento bacteriano por meio de cultura; via de parto; período de latência; colonização cervical diagnóstica por cultura de material endocervical; crescimento intra-uterino restrito, incluindo nesta variável casos com documentação ultra-sonográfica, em pelo menos duas verificações, de peso fetal abaixo do 10 o percentil 7; uso de antibiótico tanto os casos de uso terapêutico quanto profilático; e corioamnionite clínica e/ou histológica. O diagnóstico clínico de corioamnionite foi baseado na presença de temperatura axilar maior ou igual a $37,8^{\circ} \mathrm{C}$ e dois ou mais dos seguintes sinais e sintomas: taquicardia materna (maior que 100 batimentos por minuto), taquicardia fetal (160 batimentos por minuto), odor fétido do líquido amniótico e leucocitose materna (leucometria maior que 15 mil células por $\mathrm{cm}^{3}$ ) 8 .

As variáveis neonatais incluíam: idade gestacional do recém-nascido calculada pelo método de Ballard; índice de Apgar; peso ao nascer; persistência do canal arterial diagnosticado por estudo ecocardiográfico; causa do óbito dos neomortos; necessidade de manobras de ressuscitação no período neonatal e uso de ventilação mecânica.

Os desfechos estudados foram a presença de sobrevida com seqüela entre os recém-nascidos egressos de gestações que cursaram com amniorrexe e o óbito no período neonatal. No primeiro caso, foram incluídos os que tiveram os seguintes diagnósticos: hemorragia intraventricular, caracterizada pela presença de sangue na matriz germinal e/ou ventrículos laterais comprovados pela ultra-sonografia transfontanela 9; displasia broncopulmonar, considerando os casos de recém-nascidos que ainda encontravam-se dependentes de oxigenioterapia até 36 semanas de idade pós-menstrual corrigida e que apresentavam alterações radiológicas sugestivas do referido agravo 10; leucomalacia periventricular, diagnosticada pela presença de lesões típicas à avaliação da ultra-sonografia transfontanela 11 e à retinopatia da prematuridade evidenciada pela oftalmoscopia indireta demonstrando desenvolvimento anormal dos vasos retinianos para a idade em semanas do recém-nascido 12 . 
Após processo de validação interna os dados foram submetidos à análise estatística subordinada as seguintes etapas.

Inicialmente descritiva com caracterização da população estudada, distribuição dos fatores de risco já explicitados, estratificando e quantificando variáveis maternas, obstétricas e perinatais.

Seguiu-se a análise univariada, na qual as variáveis independentes (de exposição) foram confrontadas uma a uma, em tabelas $2 \mathrm{X} 2$, com os respectivos desfechos. Os testes estatísticos freqüentemente elegíveis foram o qui-quadrado corrigido ou o teste de Fisher bilateral, quando as exigências do teste qui-quadrado não puderam ser satisfeitas, considerando ambos como significativos quando $\mathrm{p} \leq 0,25$.

Por fim, procedeu-se a construção de um modelo logístico objetivando o controle de fatores de confusão, quando vigentes mais de duas variáveis com significância estatística $(p<0,05)$ na análise univariada. $\mathrm{O}$ modelo logístico seguiu o procedimento stepwise-backward manual do modelo completo. Para a elaboração dos cálculos estatísticos foram utilizados os programas Epi Info 3.4 (Centers for Disease Control and Prevention, Atlanta, Estados Unidos) e o SPSS 11.1 (SPSS Inc., Chicago, Estados Unidos).

Este estudo foi aprovado pelo Comitê de Ética em Pesquisa do IFF, tendo sido respeitadas as normas vigentes para a pesquisa em seres humanos, segundo Resolução $n^{o}$. 196/96 do Conselho Nacional de Saúde.

\section{Resultados}

Com base nos registros de prontuários do IFF foram constatadas 265 pacientes com diagnóstico de ruptura prematura pré-termo das membranas amnióticas com idade gestacional abaixo de 34 semanas entre os anos de 1998 e 2002. Desse universo amostral, apenas 173 registros constituíram o grupo de estudo por atenderem plenamente as exigências da pesquisa. Sendo excluídos 52 gestações gemelares e 40 fetos com malformações.

A idade das gestantes que integraram o estudo variou de 13 a 44 anos, com média de 26,7 \pm 7,5 anos. Quanto à paridade, $66,4 \%$ eram primíparas e 30,6\% multíparas.

A idade na qual ocorreu o evento amniorrexe prematura, considerando-se os 173 casos estudados, variou de 24 a 34 semanas, média de 28,8 $\pm 3,7$ semanas. Quando estratificados os períodos de ocorrência da amniorrexe, observaram-se as seguintes prevalências: $66 \%$ abaixo de 30 semanas enquanto os demais casos ocorreram acima de 30 semanas, correspondendo a $34 \%$ do total estudado.

Quanto ao tipo de parto, a maior parte foi transpélvico (59,5\%), seguido por cesariana em $39,3 \%$ e partos a fórcipe em $1,2 \%$ dos casos. A média de peso ao nascimento dos recém-nascidos estudados foi de $1.482,9 \pm 585,7 \mathrm{~g}$, sendo que mais de $40 \%$ apresentaram valores acima de $1.500 \mathrm{~g}$. A idade gestacional aferida pelo obstetra no momento do parto foi em média de 28,9 \pm 3,8 semanas no momento do parto. Já a aferida pelos neonatologistas pelo índice de Ballard teve como média $31,7 \% \pm 2,5$ semanas.

No concernente às variáveis maternas (Tabela 1), a prevalência de colonização cervical positiva nas gestantes foi de $30,4 \%(n=45)$. No entanto, é digno de nota que em 25 casos não foram realizadas culturas endocervicais. A infecção do trato urinário ocorreu em 17,9\% $(\mathrm{n}=31)$ das mulheres analisadas. A presença de corioamnionite clínica e/ou histológica foi observada em $67,1 \%$ das gestações envolvidas nesta pesquisa. Os fetos que tiverem diagnóstico de crescimento intra-uterino restrito durante avaliação ultra-sonográfica pré-natal perfizeram um total de $12,7 \%(\mathrm{n}=22)$ dos casos avaliados. O período de latência dito prolongado (maior que 48 horas) foi verificado em $66,5 \%(n=115)$ dos casos de amniorrexe remotas ao termo. Quanto à administração de antibiótico periparto foi constatado em $59 \%$ ( $\mathrm{n}=$ 102) dos casos.
Tabela 1

Distribuição das variáveis de natureza maternal e neonatal na população estudada.

\begin{tabular}{lcc}
\hline Variáveis maternas e neonatais & n (absoluto) & \% (relativo) \\
\hline Ventilação mecânica & 60 & 34,68 \\
Idade gestacional < 30 semanas & 88 & 18,50 \\
Peso $\leq 1.000 \mathrm{~g}$ & 42 & 23,70 \\
Peso $\leq 1.500 \mathrm{~g}$ & 95 & 44,51 \\
Reanimação cardíaca & 24 & 3,47 \\
Apgar 5o minuto < & 31 & 17,92 \\
Apgar 5o minuto $\leq 5$ & 23 & 10,40 \\
Persistência do canal arterial & 20 & 11,60 \\
Obito neonatal & 25 & 14,45 \\
UTI neonatal & 149 & 86,71 \\
Corioamnionite & 116 & 67,05 \\
Período de latência $\leq 48$ horas & 115 & 66,47 \\
Colonização cervical & 45 & 30,41 \\
Infecção do trato urinário & 31 & 17,91 \\
Infecção materna & 29 & 16,76 \\
Crescimento intra-uterino restrito & 22 & 12,72 \\
Uso de antibiótico & 102 & 58,96 \\
\hline
\end{tabular}


No tocante às variáveis neonatais (Tabela 1), os recém-nascidos submetidos à ventilação mecânica somaram 34,7\% (n = 60) da amostra em questão. A idade gestacional abaixo de 30 semanas foi verificada em $50,9 \%(n=88)$ dos neonatos do estudo. $\mathrm{O}$ peso ao nascimento abaixo de $1.000 \mathrm{~g}$ ocorreu em $24,3 \%(\mathrm{n}=42)$, enquanto os que tiveram peso igual ou menor que $1.500 \mathrm{~g}$ perfizeram 54,9\% ( $\mathrm{n}=95)$ da amostragem. O índice de Apgar $<7$ no 5 o minuto de vida foi constatado em 17,9\% ( $\mathrm{n}=31)$ dos recém-nascidos. Quando o valor do índice de Apgar de 5o minuto estudado foi reduzindo para $\leq 5$ encontramos valores em torno de $13,3 \%(n=23)$. A necessidade de reanimação cardio-pulmonar foi constatada em 13,9\% ( $n=24)$. Já a persistência do canal arterial foi observada em $11,6 \%(n=20)$ dos nascidos prematuros.

Foram diagnosticados 47 casos de recémnascidos que embora tenham sobrevivido apresentaram uma ou mais das co-morbidades definidas neste estudo como seqüela, conferindo à prevalência de sobrevida com seqüela o valor de $31,8 \%$ do referido universo amostral.

A Tabela 2 demonstra a distribuição das comorbidades que compõem o desfecho sobrevida com seqüela, com predomínio das lesões com comprometimento do sistema nervoso central, dentre estes $25 \%$ de diagnósticos foram de hemorragia intraventricular e 2,03\% de leucomalácia periventricular. As prevalências das demais foram de $14,19 \%$ e $13,51 \%$ para a displasia broncopulmonar e retinopatia da prematuridade, respectivamente, entre os sobreviventes.

A Tabela 3 apresenta a análise univariada dos fatores clínico-obstétricos e neonatais que se relacionam com o desenvolvimento das morbidades que compõem o desfecho sobrevida com seqüela. As variáveis maternas tiveram comportamentos diversos. A via de parto não apresentou associação com o desfecho em questão. O mesmo pode ser dito quanto às variáveis: infecção do trato urinário, colonização cervical e infecção materna. Já o período de latência prolongado, aqui entendido como superior a 48 horas, mostrou-se como um fator de proteção à ocorrência de dano neonatal em vigência de ruptura prematura pré-termo das membranas amnióticas [odds ratio $-\mathrm{OR}=0,42$ $(0,21-0,87) ; p=0,027]$. Após várias simulações no modelo multivariado, o mais representativo foi o demonstrado na Tabela 4, em que observamos a significância estatística das variáveis colonização cervical positiva $\mathrm{OR}=2,80(1,02-7,68)$; $\mathrm{p}=0,046]$, índice de Apgar no 5o minuto de vida $\leq 5$ [OR $=22,50(2,01-251,92) ; \mathrm{p}=0,012]$, extremo baixo peso ao nascimento $[\mathrm{OR}=6,09(1,55-23,87)$; $\mathrm{p}=0,010$ ] e a persistência do canal arterial patente $[\mathrm{OR}=6,93(1,11-43,59) ; \mathrm{p}=0,038]$.

Quanto aos casos de óbitos neonatais, a prevalência foi de 14,45\% ( $n=25)$. Em 100\% dos recém-nascidos cujo peso ao nascimento foi menor ou igual a 500g verificou-se evolução para óbito. No tocante à causa de óbito, a maior parte deuse secundariamente à prematuridade extrema (24\%), seguida pelos diagnósticos de síndrome do desconforto respiratório (20\%), hemorragia intraventricular (12\%) e sepse (12\%).

A análise univariada das variáveis maternas e neonatais cotejadas ao desfecho óbito neonatal é demonstrada na Tabela 5. Apenas a variável corioamnionite, dentre todas as de origem materna, demonstrou associação com relevância estatística com o dito desfecho [OR = 6,75 $(1,76$ 43,89); $\mathrm{p}=0,008$.

Dentre as variáveis neonatais, as de mais expressiva associação para o desfecho estudado são sem sombras de dúvidas a idade gestacional ao nascimento inferior a 30 semanas $[\mathrm{OR}=14,50$ $(3,80-94,17) ; \mathrm{p}=0,000]$, os recém-nascidos com baixo peso [OR $=5,14(1,78-18,24) ; p=0,004$ ], bem como aqueles de extremo baixo peso ao nascimento [OR $=10,17(4,00-27,50) ; \mathrm{p}=0,000$ ] e os que sofreram reanimação cardíaca $[\mathrm{OR}=$ $13,13(4,85-36,86) ; p=0,000]$. Destacam-se ainda dentro desse universo de variáveis neonatais, a ventilação mecânica $[\mathrm{OR}=8,15(3,12-23,71)$;

\begin{tabular}{lcc}
\hline Sobrevida com seqüela & Freqüência absoluta $(\mathbf{n})$ & Freqüência relativa (\%) \\
\hline Displasia broncopulmonar & 21 & 14,2 \\
Hemorragia intraventricular & 37 & 25,0 \\
Leucomalacia periventricular & 3 & 2,0 \\
Retinopatia da prematuridade & 20 & 13,5 \\
\hline
\end{tabular}


Distribuição das variáveis da população estudada, segundo análise bivariada, tendo como referência a ocorrência do desfecho sobrevida com seqüela.

\begin{tabular}{|c|c|c|c|c|}
\hline \multirow[t]{2}{*}{ Variáveis } & \multicolumn{2}{|c|}{ Sobrevida com seqüela } & \multirow[t]{2}{*}{ IC95\% } & \multirow[t]{2}{*}{$\mathrm{p}$} \\
\hline & Presente & Ausente & & \\
\hline Corioamnionite & $29 / 93$ & $18 / 55$ & $0,46-1,93$ & 0,990 \\
\hline Parto transpélvico & $31 / 90$ & $16 / 58$ & $0,67-2,88$ & 0,488 \\
\hline Período de latência $\geq 48$ horas & $24 / 96$ & $23 / 52$ & $0,21-0,87$ & 0,027 \\
\hline Colonização cervical & $15 / 36$ & $26 / 89$ & $0,75-3,88$ & 0,257 \\
\hline Infecção do trato urinário & $7 / 31$ & $38 / 117$ & $0,20-1,39$ & 0,310 \\
\hline Crescimento intra-uterino restrito & $9 / 18$ & $38 / 130$ & $0,86-6,72$ & 0,133 \\
\hline Uso de antibiótico & $22 / 57$ & $25 / 91$ & $0,81-3,37$ & 0,218 \\
\hline Ventilação mecânica & $27 / 41$ & $20 / 107$ & $3,70-18,98$ & 0,000 \\
\hline Idade gestacional $<30$ semanas & $31 / 65$ & $16 / 83$ & $1,82-8,01$ & 0,000 \\
\hline Peso $\leq 1.000 \mathrm{~g}$ & $20 / 25$ & $26 / 122$ & $5,13-46,79$ & 0,000 \\
\hline Peso $\leq 1.500 \mathrm{~g}$ & $36 / 74$ & $10 / 73$ & $2,67-13,78$ & 0,000 \\
\hline Apgar de 5 o minuto $<7$ & $6 / 13$ & $40 / 134$ & $0,60-6,56$ & 0,370 \\
\hline Apgar de 5 ㅇ minuto $\leq 5$ & $6 / 8$ & $41 / 140$ & $1,45-53,20$ & 0,021 \\
\hline Persistência do canal arterial & $13 / 15$ & $34 / 133$ & $4,46-126,39$ & 0,000 \\
\hline Reanimação cardíaca & $9 / 11$ & $38 / 137$ & $2,60-81,25$ & 0,000 \\
\hline
\end{tabular}

Tabela 4

Análise multivariada dos fatores mais significativamente relacionados com o desfecho sobrevida com seqüela. Modelo final.

\begin{tabular}{|c|c|c|c|c|}
\hline Variáveis & $\beta$ & OR & IC95\% & $\mathrm{p}$ \\
\hline Colonização cervical & 1,028 & 2,80 & $1,02-7,68$ & 0,046 \\
\hline Apgar de 5 o minuto $\leq 5$ & 3,110 & 22,50 & $2,01-251,92$ & 0,012 \\
\hline Peso $\leq 1.000 \mathrm{~g}$ & 1,807 & 6,09 & $1,55-23,87$ & 0,010 \\
\hline Persistência do canal arterial & 1,941 & 6,93 & $1,11-43,59$ & 0,038 \\
\hline Ventilação mecânica & 0,926 & 2,52 & $0,81-7,87$ & 0,110 \\
\hline
\end{tabular}

$\mathrm{p}=0,000]$, índice de Apgar de 5o minuto $<7$ [OR $=25,53(9,22-77,15) ; \mathrm{p}=0,000]$ e índice de Apgar de 5 o minuto $\leq 5$ [OR = 11,37 (3,86-34,67); $\mathrm{p}=0,000]$. Na modelagem multivariada final, conforme demonstrado na Tabela 6, observase uma forte associação dos casos de evolução letal com a variável corioamnionite [OR $=11,24$ $(11,32-95,73) ; p=0,027]$. Nota-se ainda significância estatística quanto à necessidade de reanimação cardíaca [OR =6,61 $(1,80-24,24) ; \mathrm{p}$ $=0,004]$, peso ao nascimento inferior a $1.000 \mathrm{~g}$ [OR = 3,66 (1,15-11,66); $\mathrm{p}=0,028]$ e índice de Apgar de 5o minuto $\leq 5$ [OR = 14,32 (3,35-61,32); $\mathrm{p}=0,000]$. Já a necessidade de ventilação mecânica falhou em demonstrar associação com o óbito neonatal.

\section{Discussão}

Diante dos grandes avanços tecnológicos na área de cuidados intensivos neonatais torna-se cada vez mais freqüente a sobrevivência de recém-nascidos com idades gestacionais mais tenras, bem como com pesos ao nascimento mais baixos. Em torno de um terço desses prematuros é oriundo de gestações que tiveram como intercorrência a amniorrrexe prematura remota ao termo, e pelo menos $20 \%$ destas gestações foram encerradas por decisão médica, seja por risco materno ou fetal 13,14 .

Em nossa amostra foram observados 25 casos de óbito no período neonatal, correspondendo a aproximadamente $14,5 \%$ do total de casos 
Distribuição das variáveis da população estudada, segundo análise bivariada, tendo como referência a ocorrência de óbito neonatal.

\begin{tabular}{lcccc}
\hline Variáveis & \multicolumn{2}{c}{ Óbito neonatal } & IC95\% & \\
& Presente & Ausente & \\
& & $2 / 57$ & $1,76-43,89$ & 0,008 \\
Corioamnionite & $23 / 116$ & $6 / 58$ & $0,66-4,94$ & 0,389 \\
Período de latência $\geq 48$ horas & $19 / 115$ & $12 / 101$ & $1,69-4,81$ & 0,300 \\
Colonização cervical & $9 / 45$ & $11 / 102$ & $0,85-4,89$ & 0,154 \\
Uso de antibiótico & $14 / 71$ & $10 / 68$ & $0,41-2,37$ & 0,885 \\
Parto transpélvico & $15 / 105$ & $21 / 157$ & $0,37-4,29$ & 0,835 \\
Crescimento intra-uterino restrito & $4 / 22$ & $6 / 113$ & $3,12-3,71$ & 0,000 \\
Ventilação mecânica & $19 / 60$ & $2 / 85$ & $3,80-4,17$ & 0,000 \\
Idade gestacional $<30$ semanas & $23 / 88$ & $8 / 131$ & $4,00-7,50$ & 0,000 \\
Peso $\leq 1.000 g$ & $17 / 42$ & $4 / 78$ & $1,78-8,24$ & 0,004 \\
Peso $\leq 1.500 g$ & $21 / 95$ & $7 / 141$ & $9,22-7,15$ & 0,000 \\
Apgar 5o minuto $\leq 7$ & $18 / 31$ & $15 / 154$ & $3,86-4,67$ & 0,000 \\
Apgar 5o minuto $\leq 5$ & $10 / 18$ & $20 / 153$ & $0,65-6,61$ & 0,276 \\
Persistência do canal arterial & $5 / 20$ & $12 / 149$ & $4,85-6,86$ & 0,000 \\
Reanimação cardíaca & $13 / 24$ & &
\end{tabular}

Tabela 6

Análise multivariada dos fatores mais significativamente relacionados com o óbito neonatal. Modelo final.

\begin{tabular}{lcccc}
\hline Variáveis & $\beta$ & OR & IC95\% & P \\
\hline Corioamnionite & 2,419 & 11,24 & $1,32-95,73$ & 0,027 \\
Apgar de 5o minuto $\leq 5$ & 2,662 & 14,32 & $3,35-61,32$ & 0,000 \\
Peso $\leq 1.000 \mathrm{~g}$ & 1,297 & 3,66 & $1,15-11,66$ & 0,028 \\
Ventilação mecânica & 1,054 & 2,87 & $0,70-11,78$ & 0,143 \\
Reanimação cardíaca & 1,890 & 6,61 & $1,80-24,24$ & 0,004 \\
\hline
\end{tabular}

estudados. A principal causa de morte apontada pelo estudo foi a prematuridade extrema, reforçando a importância deste agravo no incremento das taxas de óbito neonatal, sobretudo entre egressos de gestações com ruptura prematura pré-termo das membranas amnióticas. Bang et al. 15, em estudo sobre a estimativa percentual de causa de óbitos neonatais globais para o ano 2000, demonstraram que em torno de $28 \%$ dos casos resultaram de causas correlacionadas à prematuridade, $36 \%$ de graves infecções neonatais (que incluíam sepses/pneumonia, tétano, diarréia em $26 \%$, $7 \%$ e $3 \%$ dos casos, respectivamente) e 23\% secundárias a complicações hipóxico-isquêmicas. Dos 14\% restantes apenas $7 \%$ foram consistentemente relacionadas a anomalias congênitas.
Nos Estados Unidos da América, a taxa de sobrevida varia entre $20 \%$ e $30 \%$ quando a idade gestacional dos neonatos estudados está entre 22 e 23 semanas, com elevada incidência de alterações no desenvolvimento neurológico ${ }^{16}$. Spitz et al. 17 constataram, por meio de estudo multicêntrico, que a sobrevida global de recém-nascidos de mães com ruptura prematura pré-termo das membranas amnióticas com idades gestacionais inferiores a 26 semanas foi de $35 \%$, e que mais de $70 \%$ das crianças que sobreviveram tiveram um bom rendimento escolar.

Quanto ao período de latência superior a 48 horas parece haver maior chance de infecção materna e fetal (principalmente por infecção ascendente do trato genital inferior), e a exposição a outras complicações antenatais como: a data 
provável do parto (DPP), compressão funicular secundária ao inóspito ambiente de oligodramnia e prolapso de cordão com maiores chances de comprometimento neonatal futuro ${ }^{1}$. No entanto, nesta pesquisa à luz da análise univariada, constatou-se que o período de latência maior que 48 horas teve efeito protetor sobre a ocorrência de seqüela entre os neonatos sobreviventes $[\mathrm{OR}=0,42(0,21-0,87) ; \mathrm{p}=0,027]$, sendo que $34 \%(n=59)$ dos casos com período de latência prolongado foram observados em idade gestacional abaixo de 30 semanas e $32,37 \%(n=56)$ acima de 30 semanas. Resultado semelhante foi encontrado por Pasquier et al. 18 em estudo de coorte prospectivo no qual avaliaram mais de 470 mulheres com ruptura prematura pré-termo das membranas amnióticas ocorrida entre a 24a e a 33a semanas de gestação, destas 301 (63\%) tiveram período de latência maior que 48 horas. A taxa de mortalidade foi maior entre neonatos com idade gestacional inferior a 30 semanas com período de latência mais curto $(16,3 \%$ versus $7,3 \%$; $<$ 0,001), no entanto, quando analisados os neonatos com idade ao nascimento maior que 30 semanas com período de latência menor que 48 horas, a taxa de mortalidade mostrou-se mais baixa. Já a incidência de dano neurológico em dois anos de acompanhamento desses pacientes não mostrou alteração quando comparadas as diferentes durações dos períodos de latência analisados. Acreditamos que o período de latência prolongado possa ter efeito benéfico sobre o resultado neonatal se associado à ocorrência de ruptura prematura pré-termo das membranas amnióticas em idades gestacionais abaixo de 30 semanas, possibilitando maior desenvolvimento estrutural e funcional de órgãos e sistemas fetais, reduzindo os insultos correlacionados à prematuridade, viabilizando o uso corticóide e antibiótico antenatal.

Muitos estudos têm sinalizado que os fatores etiopatogênicos relacionados à ocorrência da amniorrexe prematura, principalmente de natureza infecciosa/inflamatória, possam de forma independente funcionar como elementos adicionais à própria prematuridade, no tocante à ocorrência de dano neonatal 14,19,20. Os mecanismos fisiopatológicos responsáveis pelo trabalho de parto prematuro e pela ruptura prematura pré-termo das membranas amnióticas, nesse caso, envolvem uma resposta inflamatória sistêmica, com aumento de citocinas, prostaglandinas, colagenases e mataloproteinases 21,22. Segundo Goldenberg et al. 23, as evidências apontam para o fato de que a maioria dos partos prematuros seja causada por infecções bacterianas do corioamnio, com origem no trato genital inferior. Muitas das morbidades ligadas ao nascimento pré-termo podem isoladamente ou em associação aumentar o tempo de permanência desses recém-nascidos em unidades de terapia intensiva, majorar a taxa de reinternação por complicações futuras, bem com gastos públicos na saúde relacionados a manutenções dos que sobrevivem aos agravos, mas não livre de seqüela.

Neste estudo os processos infecciosos/inflamatórios pesquisados foram basicamente a presença de corioamnionite clínica e/ou histológica e a colonização cervical positiva em avaliação pré-natal. Ambas as variáveis em questão mostraram-se como fator de risco para má evolução neonatal, a primeira relacionando ao óbito neonatal (Tabela 6) e à colonização cervical a presença de seqüela entre os sobreviventes (Tabela 4).

A ocorrência de corioamnionite é uma séria complicação da gravidez e representa um argumento contra a conduta conservadora na presença de ruptura prematura pré-termo das membranas amnióticas, estando associada ao dano neonatal, óbito fetal e neonatal, assim como o nascimento pré-termo e ao baixo peso ao nascimento 5,24. A taxa de infecção é diretamente proporcional à duração do período de latência, aumentando após as primeiras 24 horas e sendo pelo menos dez vezes maior após 72 horas de amniorrexe 23. Muitos estudos ainda atribuem essa relação direta da corioamnionite com os desfechos óbito e seqüela entre os neonatos sobreviventes ao agravo, ao desencadeamento de uma resposta inflamatória sistêmica fetal que conduz à importante disfunção orgânica intermediada por uma complexa rede de citocinas pró-inflamatórias e moléculas de glicoproteínas de alto e baixo pesos moleculares produzidas por monócitos/macrófagos 3,25,26. Elimian et al. 27 estudaram as placentas de 1.260 recém-nascidos com peso igual ou inferior a $1.750 \mathrm{~g}$, destas 527 apresentavam sinais histológicos de corioamnionite, e neste subgrupo também encontraram maior taxa de mortalidade neonatal, bem como maior prevalência de morbidade neonatal do que no grupo cujas placentas não exibiam sinais inflamatórios.

Ogunyemi et al. 28 analisaram 774 neonatos com idade gestacional entre 24 e 32 semanas ao nascimento. A corioamnionite histológica foi identificada em $46 \%$ dos casos com ruptura prematura pré-termo das membranas amnióticas e $45 \%$ dos casos com parto prematuro, sendo apontados como fator de risco para hemorragia intraventricular (71\% dos casos versus $23 \%$ dos controles; $\mathrm{OR}=2.20 ; \mathrm{p}=0,001$ ), displasia broncopulmonar (26\% dos casos versus $15 \%$ dos controles; $\mathrm{OR}=2,00 ; \mathrm{p}=0,001)$, retinopatia da prematuridade (36\% dos casos versus $24 \%$ dos controles; $\mathrm{OR}=1,80 ; \mathrm{p}=0,0001$ ) e óbito neona- 
tal $(12 \%$ dos casos versus $7 \%$ dos controles; $\mathrm{OR}=$ 2,00; $\mathrm{p}=0,012$ ).

Quanto ao uso de antibiótico na vigência de ruptura prematura pré-termo das membranas amnióticas, a presente pesquisa não demonstrou significância estatística sobre a morbimortalidade neonatal. Kenyon at al. 29, de forma distinta, em revisão sistemática da literatura do uso de antibiótico em ruptura prematura pré-termo das membranas amnióticas versus o uso de placebo em 14 trabalhos, encontraram associação entre o uso de antibiótico e uma redução da infecção materna e a corioamnionite. Também foi observada importante redução da mortalidade perinatal (intervalo de $95 \%$ de confiança - IC95\%: 0,75-1,11), infecção neonatal (IC95\%: $0,52-0,85)$, anormalidades na avaliação ultrasonográfica cerebral (IC95\%: 0,68-0,99), hemocultura neonatal positiva (IC95: 0,60-0,93), uso de surfactante (IC95\%: 0,72-0,96), oxigenioterapia (IC95\%: 0,81-0,96). Resultado semelhante foi apontado por Mercer \& Lewis 8 com aumento do período de latência de 2,9 para 6,1 dias, em média, com uso de antibioticoterapia de amplo espectro em gestações pré-termo associado à ruptura prematura pré-termo das membranas amnióticas. Possivelmente a discordância entre o resultado encontrado nesta pesquisa e a tendência atual de uso de antibioticoterapia na abordagem conservadora da ruptura prematura pré-termo das membranas amnióticas seja fruto da multifatorialidade dos agravos que podem levar a dano e óbito no feto pré-termo na presença de ruptura prematura pré-termo das membranas amnióticas, ao reduzido número de casos que compôs o universo amostral, bem como a não descriminação dos casos em que o antibiótico foi usado de forma profilática ou terapêutica.

Dentre as variáveis neonatais, o peso ao nascimento e a idade gestacional destacam-se como relevantes fatores a serem levados em consideração no manejo clínico e avaliação prognóstica quanto à sobrevida e ao dano pós-natal 30. Para muitos os principais agravos perinatais relacionados à ruptura prematura pré-termo das membranas amnióticas são determinados pela imaturidade de órgãos e sistemas fetais, concluindo desta forma que os efeitos da prematuridade seriam o principal fator responsável pelo dano neonatal entre as gestações que cursaram com amniorrexe prematura pré-termo 31 . Chard et al. 32 , em estudo populacional sobre os fatores de risco para morte neonatal que envolveu uma amostra de aproximadamente 7 mil casos de neonatos pré-termo, concluíram que o baixo peso ao nascimento demonstrou associação positiva para o aumento da mortalidade neonatal.
Shankaran et al. 33 realizaram um estudo multicêntrico prospectivo com o objetivo de estabelecer o risco de morte neonatal nas primeiras 12 horas de vida entre recém-nascidos de muito baixo peso. Aqueles que foram a óbito precocemente apresentaram menores índices de Apgar, peso ao nascimento mais baixo com aumento do risco em quase duas vezes a cada menos $50 \mathrm{~g}$ (IC95\%: 1,2-1,4), menor índice de entubação e uso de ventilação mecânica. Destacam-se ainda outros fatores apontados pelo estudo como de importante risco para morte nas primeiras 12 horas de vida: ausência de uso de surfactante, sexo masculino, ausência de administração de corticóide antenatal, e idade gestacional mais prematura.

Dessa forma, a idade gestacional e o peso ao nascimento estão consistentemente relacionados ao aumento na incidência da mortalidade e algumas morbidades relacionadas à prematuridade, tais como: leucomalácia periventricular, retinopatia da prematuridade, hemorragia intraventricular, displasia broncopulmonar, persistência do canal arterial, sepses neonatal, enterocolite necrosante, tendo comportamento inversamente proporcional a estes 1,5.

Quanto à avaliação dos danos causados pela hipóxia na população estudada foi utilizado como marcador o índice de Apgar. Os recém-nascidos, no presente estudo, que obtiveram Apgar $<7$ tinham demonstrado risco aumentado para óbito [OR = 25,53 (9,22-77,15); $\mathrm{p}=0,000]$, não houve correlação estatística entre o índice de Apgar e a presença de dano neonatal. Tal achado pode ser reflexo do fato de o índice de Apgar não ser um bom indicador para asfixia neonatal em prematuros, principalmente aqueles com muito baixo peso. Segundo Snyder \& Clohetyr 34 , as causas de Apgar com baixos valores sem concomitância de estados asfíxicos são: insultos metabólicos, trauma, depressão por analgésicos ou anestesia materna, distúrbios neuromusculares ou malformações pulmonares. Além disso, ainda que um baixo escore de Apgar possa sinalizar um neonato deprimido, ele não indica a duração ou gravidade do insulto específico ou a resposta adaptativa fetal.

Quando abaixamos o ponto de corte do Apgar de 5 o minuto para valores menores do que 5 e correlacionamos aos desfechos estudados encontramos relevância estatística na análise univariada [óbito neonatal - OR = 11,37 (3,86$34,67) ; \mathrm{p}=0,000$; sobrevida com seqüela $-\mathrm{OR}=$ 7,14 (1,45-53,20); $\mathrm{p}=0,021$.

Semelhante aos resultados encontrados, Moster et al. 35 em estudo de coorte populacional estudaram mais de 235 mil recém-nascidos com peso menor ou igual a $2.500 \mathrm{~g}$, comparan- 
do os com Apgar de 5o minuto entre 0 e 3 com aqueles cujo referido escore ficou entre 7 e 10 . Concluíram que risco de morte neonatal e dano cerebral era maior quando o Apgar de 1o minuto assim como o de quinto minuto ficavam entre 0 e 3, aumentando em 642 vezes e 145 vezes, respectivamente, o risco dos referentes agravos. Caravale et al. 36 apontam o índice Apgar de $5 \underline{0}$ minuto abaixo de 5 como um preditor para risco de comprometimento neurológico fetal $(\mathrm{p}=$ $0,0001)$, principalmente a quadro convulsivo no período neonatal.

A presença de persistência do ducto arterial, agravo de íntima correlação com a prematuridade, foi constatada em 11,56\% ( $n=20)$ dos recém-nascidos prematuros que integram o estudo. Desses, $85 \%$ tinham idade gestacional inferior a 30 semanas e $75 \%$ tinham peso inferior a $1.000 \mathrm{~g}$ ao nascimento, estabelecendo importante associação desse agravo com a presença de seqüelas entre os sobreviventes na análise univariada [OR = 18,51 (4,46-126,39); $\mathrm{p}=0,000]$. Contudo, para o desfecho óbito neonatal, não foi observada relação estatisticamente significativa.

Os achados da pesquisa em relação à persistência do ducto arterial estão em sintonia com a literatura científica que estabelece a maior prevalência do referido insulto em recém-nascidos de baixo peso ao nascimento e idades gestacionais mais remotas ao termo ${ }^{37}$. Para Baptista et al. 38 essa condição está associada a maiores freqüências de terapia com surfactante, ventilação mecânica, displasia broncopulmonar, enterocolite necrosante, e hemorragia intraventricular. Bassan et al. 39 ainda destacam a persistência do canal arterial como um fator independente de risco para dano cerebral em recém-nascidos pré-termos e com baixo peso, correlacionando o achado com o decréscimo do fluxo sangüíneo diastólico e, pela veia cava superior, conduzindo a distúrbios perfusionais e de oxigenação cerebral.

\section{Conclusões}

Os resultados da análise multivariada descrevem de forma apurada o perfil dos recém-nascidos oriundos de gestações que tiveram como intercorrência a ruptura pré-termo das membranas amnióticas, que ao agregarem fatores de risco à sua própria condição de prematuro, acabam por evoluir para óbito mesmo em detrimento de todo o aparato tecnológico e terapêutico a eles oferecido. Em geral, prematuros extremos, com extremo baixo peso, asfíxicos graves e aqueles com comprometimento infeccioso e/ou inflamatório intra-uterino, como demonstrado em muitos trabalhos 3,24 .

No concernente aos recém-nascidos que sobreviveram ao agravo, mas não livres de seqüelas, mais uma vez o muito baixo peso ao nascer, a história de asfixia intra-uterina ou periparto e a associação à plausível contaminação infecciosa intra-uterina aparecem como fatores de suma relevância na determinância de danos a múltiplos órgãos e sistemas fetais. Percebe-se que o dano adicional causado pelo agravo hipóxico acrescido à prematuridade majora de forma expressiva as taxas de morbimortalidade neonatal 39.

A análise da mortalidade neonatal e das morbidades pós-natais associadas ao nascimento pré-termo decorrente da ruptura prematura prétermo nas membranas amnióticas e de seus fatores de riscos é essencial para identificar possíveis impactos de mudanças sociais e econômicas e dos avanços, e eventuais retrocessos, da cobertura e da qualidade dos serviços de assistência pré-natal e nosocomial. Embora a mortalidade e a morbidade neonatal sejam resultantes de uma estreita e complexa relação entre as variáveis biológicas como amplamente discutido neste artigo, é importante ressaltar que elementos sócio-econômicos e culturais, bem como fatores assistenciais à saúde, devem fazer parte de estratégias de ação rumo à redução das expressivas taxas de morbimortalidade neonatal dos países em desenvolvimento. 


\section{Resumo}

O objetivo foi analisar os fatores associados ao óbito e sobrevida com seqüela em neonatos egressos de gestações que cursaram com amniorrexe prematura. Estudo observacional do tipo coorte histórica analisou prontuários de pacientes que evoluíram com quadro de amniorrexe prematura $\leq 34$ semanas de gestação. As variáveis foram cotejadas com os desfechos óbito neonatal e sobrevida com seqüela. Em ambos os grupos os dados foram submetidos à análise bivariada, sendo os valores que exibem significância estatística submetidos à regressão logística. No modelo multivariado final em relação ao óbito fetal tiveram significância estatística: corioamnionite; índice de Apgar $\leq 5$ no 5 o minuto; peso ao nascimento $\leq 1.000 \mathrm{~g}$; e reanimação cardiopulmonar. Quanto à presença de seqüela entre os sobreviventes destacam se: a colonização cervical; persistência do canal arterial; índice de Apgar $\leq 5$ no 5 o minuto; e peso ao nascimento $\leq 1.000 \mathrm{~g}$. Processos infecciosos, extremo baixo peso ao nascer e asfixia periparto são as principais variáveis ligadas aos desfechos estudados entre neonatos egressos de gestações que cursaram com amniorrexe prematura.

Ruptura Prematura de Membranas Fetais; Mortalidade Neonatal; Morbidade

\section{Referências}

1. Mercer BM. Preterm premature rupture of the membrane. Obstet Gynecol 2003; 101:178-93.

2. Furman B, Shoham-Vardi I, Bashiri A, Erez O, Mazor M. Clinical significance and outcome of preterm prelabor rupture of membranes: population-band study. Eur J Obstet Gynecol Reprod Biol 2000; 92:209-16.

3. Romero R, Espinoza J, Gonçalves LF, Kusanovic JP, Friel LA, Nien JK. Inflammation in preterm and term labour and delivery. Semin Fetal Neonatal Med 2006; 11:317-26.

4. Watts JL, Saigal S. Outcome of extreme prematurity: as information increases so do the dilemmas. Arch Dis Child Fetal Neonatal Ed 2006; 91:F221-5.

5. Dammann O, Leviton A, Gappa M, Dammann CE. Lung and brain damage in preterm newborns, and their association with gestational age, prematurity subgroup, infection/inflammation and long term outcome. BJOG 2005; 112 Suppl 1:4-9.

6. Hnat MD, Mercer BM, Thurnau G, Goldenberg R, Thom EA, Meis PJ, et al. Perinatal outcomes in women with preterm rupture of membranes between 24 and 34 weeks of gestation and a history of vaginal bleeding. Am J Obstet Gynecol 2005; 193:164-8.

\section{Colaboradores}

Os autores participaram de todas as etapas da pesquisa e da redação do artigo.
7. Fang S. Management of preterm infants with intrauterine growth restriction. Early Hum Dev 2005; 81:889-900.

8. Mercer BM, Lewis R. Preterm labor and preterm premature rupture of the membranes. Diagnosis and management. Infect Dis Clin North Am 1997; 11:775-82.

9. Papile LA, Burstein J, Burstein R, Koffler H. Incidence and evolution of subependymal and intraventricular hemorrhage: a study of infants with birth weight less than 1,500gm. J Pediatr 1978; 92:529-34.

10. Oliveira RG. Persistência no canal arterial no recém nascido prematuro. In: Oliveira RG, organizador. Black Book: manual de referências de pediatria. v. 1. Belo Horizonte: s.e.; 2002. p. 446-7.

11. Hamrick SE, Miller SP, Leonard C, Glidden DV, Goldenstein R, Ramaswany V, et al. Trends in severe brain injury and developmental outcome in premature newborn infants: the role of cystic periventricular leukomalacia. J Pediatr 2004; 145:593-9.

12. Anderson CL, Stewart JE. Problemas auditivos e oftalmológicos. In: Cloherty JP, Stark AP, organizadores. Manual de neonatologia. Rio de Janeiro: Editora Medsi; 2000. p. 680-7. 
13. Andrews WW, Sibai BM, Thom EA, Dudley D, Ernest JM, Mcvellis D, et al. Randomized clinical trial to prevent spontaneous preterm delivery in the fetal fibronectin-positive women. Obstet Gynecol 2003; 101:847-55.

14. Goldenberg RL, Mwatha A, Read JS, Adeniyi-Jones S, Sinkala M, Msmanga G, et al. The HPTN 024 Study: the efficacy of antibiotics to prevent chorioamnionitis and preterm birth. Am J Obstet Gynecol 2006; 194:650-61.

15. Bang A, Reddy MH, Deshmukh MD. Child mortality in Mahararstra. Econ Polit Wkly 2002; 37: 4947-65.

16. Hack M, Fanaroff AA. Outcomes of children of extremely low birth weight and gestational age in the 1990's. Early Hum Dev 1999; 53:193-218.

17. Spitz B, Vossen C, Devlieger R, Van Assche FA. Rupture of membranes before 26 weeks of gestation: outcome of 148 consecutive cases. J Perinat Med 1999; 27:451-7.

18. Pasquier JC, Bujold E, Rabilloud M, Picaud JC, Ecochard R, Claris O, et al. Effect of latency period after premature rupture of membranes on 2 years infant mortality (DOMINOS study). Eur J Obstet Gynecol Reprod Biol 2007; 135:21-7.

19. Wharton KN, Pinar H, Stonestreet BS, Tucker R, McLean K, Wallach M, et al. Severe umbilical cord inflammation- a predictor of periventricular leukomalacia in very low birth weight infants. Early Hum Dev 2004; 77:77-87.

20. Shim SS, Romero R, Hong JS, Park CW, Jun JK, Kim $\mathrm{BI}$, et al. Clinical significance of intra-amniotic inflammation in patients with preterm premature rupture of membranes. Am J Obstet Gynecol 2004; 191:1339-45.

21. Hagberg H, Mallard C, Jacobsson B. Role of cytocines in preterm labour and brain injury. BJOG 2005; 112 Suppl 1:16-8.

22. Moore RM, Mansour JM, Redline RW, Mercer BM, Moore JJ. The physiology of fetal membrane rupture: insight gained from the determination of physical properties. Placenta 2006; 27:1037-51.

23. Goldenberg RL, Houth JC, Andrews WW. Intrauterine infection and preterm delivery. N Engl J Med 2002; 342:1500-7.

24. Kallapur SG, Ikegami M. Physiological consequences of intrauterine insults. Paediatr Respir Rev 2006; 7:110-6.

25. Ng PC, Li K, Wong RP, Chui K, Wong E, Li G, et al. Proinflammatory and anti-inflammatory cytokine responses in preterm infants with systemic infections. Arch Dis Child Fetal Neonatal Ed 2003; 88: F209-13.

26. Cornette L. Fetal and neonatal inflammatory response and adverse outcome. Semin Fetal Neonatal Med 2004; 9:459-70.
27. Elimian A, Verma U, Beneck D, Cipriano R, Visintainer P, Tejani N. Histologic chorioamnionitis, antenatal steroids, and perinatal outcomes. Obstet Gynecol 2000; 96:333-6.

28. Ogunyeni D, Murillo M, Jackson U, Hunter N, Alperson B. The relationship between placental histopathology findings and perinatal outcome in preterm infants. J Matern Fetal Neonatal Med 2003; 13:102-9.

29. Kenyon S, Boulvain M, Neilson J. Antibiotics for preterm rupture of the membranes: a systematic review. Obstet Gynecol 2004; 104(5 Pt 1):1051-7.

30. Dorling J, D'Amore A, Salt A, Seward A, Kaptoge S, Holliday S, et al. Data collection from very low birth weight infants in a geographical region: methods costs, and trends in mortality, admission rates, and resources utilization over a five fear period. Early Hum Dev 2006; 82:117-24.

31. Furman B, Shoham-Vardi I, Bashiri A, Erez O, Mazor M. Preterm premature rupture of membranes is not an independent risk factor for neonatal morbidity. J Matern Fetal Med 2001; 10:107-11.

32. Chard T, Penney G, Chalmers J. The risk of neonatal death in relation to birth weight and maternal hypertensive disease in infants born at 24-32 weeks. Eur J Obstet Gynecol Reprod Biol 2001; 95:114-8.

33. Shankaran S, Fanaroff AA, Wright LL, Stevenson DK, Donovan EF, Ehrenkranz RA, et al. Risk factors for early death among extremely low-birth-weight infants. Am J Obstet Gynecol 2002; 186:796-802.

34. Snyder EV, Cloherty JP. Asfixia perinatal. In: Clolert JP, Stark AR, organizadores. Manual de neonatologia. Rio de Janeiro: Editora Medsi; 2000. p. 543-63.

35. Moster D, Lie RT, Irgens LM, Bjerkedal T, Markestad T. The association of Apgar score with subsequent death and cerebral palsy: a population-based study in term infants. J Pediatr 2001; 138:798-803.

36. Caravale B, Allemand F, Libenson MH. Factors predictive of seizures and neurologic outcome in perinatal depression. Pediatr Neurol 2003; 29:18-25.

37. Shimada S, Kasai T, Hoshi A, Murata A, Chida S. Cardiocirculatory effects of patent ductus arteriosus in extremely low-birth-weight infants with respiratory distress syndrome. Pediatr Int 2003; 45:255-262.

38. Baptista MJ, Correia-Pinto J, Areias JC, Guimarães H. Patent ductus arteriosus in neonatal intensive care. Rev Port Cardiol 1999; 18:1095-100.

39. Bassan H, Feldman HA, Limperopoulos C, Benson CB, Ringer AS, Veracruz E, et al. Periventricular hemorrhagic infarction: risk factors and neonatal outcome. Pedriatr Neurol 2006; 35:85-92.

Recebido em 12/Nov/2007

Versão final reapresentada em 07/Abr/2008

Aprovado em 16/Abr/2008 